\title{
Holographic Near-Eye Displays Based on Overlap-Add Stereograms
}

\author{
NITISH PADMANABAN, Stanford University \\ YIFAN PENG, Stanford University \\ GORDON WETZSTEIN, Stanford University
}

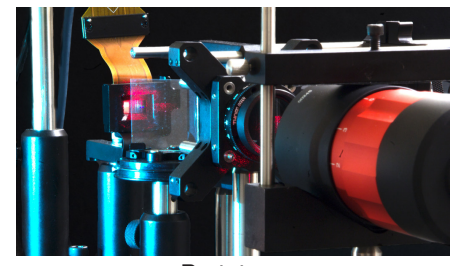

Prototype

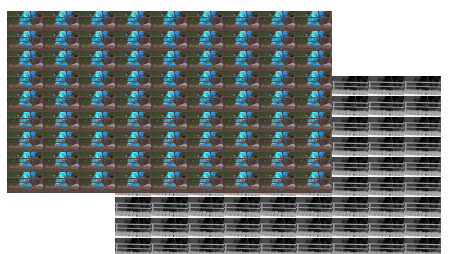

Light Field + Depth

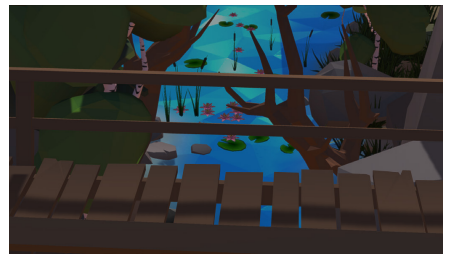

OLAS Amplitude

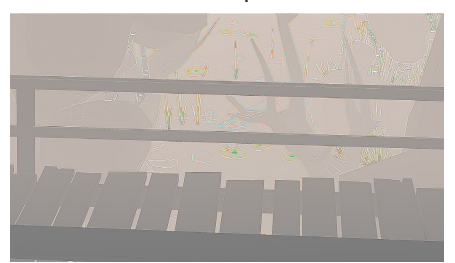

OLAS Phase
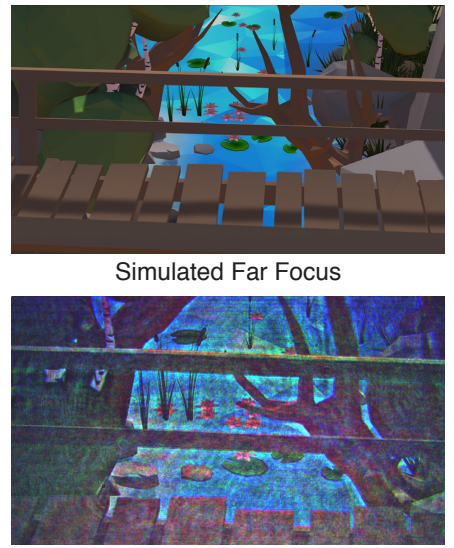

Captured Far Focus

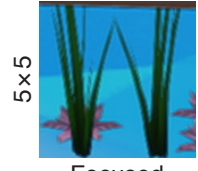

Focused

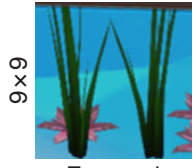

Focused

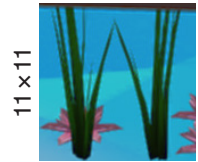

Focused

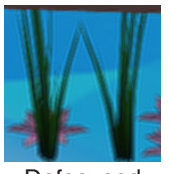

Defocused
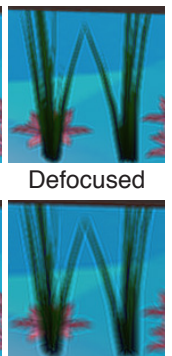

Defocused

Fig. 1. Light fields and holograms are two different methods of encoding a 3D scene, one based on the ray optics model and the other on wave optics. We develop the overlap-add stereogram (OLAS) algorithm, which is a type of holographic stereogram. The OLAS uses overlapping hogels to encode the view-dependent lighting effects of a light field (left, bottom) into a hologram. Seen above is the calculated amplitude and phase for a given light field (center left, phase range clamped to $\pm \frac{\pi}{6}$ for visualization). This complex-valued wavefront is propagated to simulate far focus (center right, top), and also captured (center right, bottom) after being displayed on a near-eye display (left, top). Finally, we show that the OLAS method produces image quality that is independent of the hogel size used (right, hogel sizes labeled), assuming the underlying light field is not undersampled angularly. This represents a significant improvement over typical holographic stereogram algorithms, which require per-object tuning of hogel size for optimal resolution.

Holographic near-eye displays are a key enabling technology for virtual and augmented reality (VR/AR) applications. Holographic stereograms (HS) are a method of encoding a light field into a hologram, which enables them to natively support view-dependent lighting effects. However, existing HS algorithms require the choice of a hogel size, forcing a tradeoff between spatial and angular resolution. Based on the fact that the short-time Fourier transform (STFT) connects a hologram to its observable light field, we develop the overlap-add stereogram (OLAS) as the correct method of "inverting" the light field into a hologram via the STFT. The OLAS makes more efficient use of the information contained within the light field than previous HS algorithms, exhibiting better image quality at a range of distances and hogel sizes. Most remarkably, the OLAS does not degrade spatial resolution with increasing hogel size, overcoming the spatio-angular resolution tradeoff that previous HS algorithms face. Importantly, the optimal hogel size of previous methods typically varies with the depth of every object in a scene,

Authors' addresses: Nitish Padmanaban, Electrical Engineering Department, Stanford University, nit@stanford.edu; Yifan Peng, Electrical Engineering Department, Stan ford University, evanpeng@stanford.edu; Gordon Wetzstein, Electrical Engineering Department, Stanford University, gordon.wetzstein@stanford.edu.

Permission to make digital or hard copies of all or part of this work for personal or classroom use is granted without fee provided that copies are not made or distributed for profit or commercial advantage and that copies bear this notice and the full citation on the first page. Copyrights for components of this work owned by others than the author(s) must be honored. Abstracting with credit is permitted. To copy otherwise, or republish, to post on servers or to redistribute to lists, requires prior specific permission and/or a fee. Request permissions from permissions@acm.org.

(C) 2019 Copyright held by the owner/author(s). Publication rights licensed to ACM.

0730-0301/2019/11-ART214 \$15.00

https://doi.org/10.1145/3355089.3356517 making the OLAS not only a hogel size-invariant method, but also nearly scene independent. We demonstrate the performance of the OLAS both in simulation and on a prototype near-eye display system, showing focusing capabilities and view-dependent effects.

CCS Concepts: • Hardware $\rightarrow$ Displays and imagers; • Computing methodologies $\rightarrow$ Computer graphics.

Additional Key Words and Phrases: computational displays, holography, light fields, virtual reality, augmented reality

\section{ACM Reference Format:}

Nitish Padmanaban, Yifan Peng, and Gordon Wetzstein. 2019. Holographic Near-Eye Displays Based on Overlap-Add Stereograms. ACM Trans. Graph. 38, 6, Article 214 (November 2019), 13 pages. https://doi.org/10.1145/3355089. 3356517

\section{INTRODUCTION}

Virtual or augmented reality (VR/AR) systems have widespread applications in entertainment, education, communication, training, behavioral therapy, and basic vision research. In all of these applications, the primary interface between the user and the wearable computer is a near-eye display. Although consumer devices such as the Microsoft HoloLens, Magic Leap One, HTC Vive, and Oculus Rift have enabled unprecedented user experiences, the underlying display technology must be significantly improved to optimize device form factors, weight, field of view, eyebox, focusing capabilities as well as image resolution, brightness and contrast. One of the most 
Table 1. Algorithm Comparison

\begin{tabular}{|r|c|c|c|c|c|}
\cline { 2 - 6 } \multicolumn{1}{c|}{} & LF & HS & PAS & OLAS & Fresnel \\
\hline Resolution & low & low & low & high & high \\
\hline Depth of field & low & low & medium & high & high \\
\hline Computation & low & medium & medium & med.-high & high \\
\hline View-dep. effects & yes & yes & yes & yes & sometimes \\
\hline Input data & - & LF & LF-D & LF-D & RGB-D \\
\hline
\end{tabular}

Fresnel holograms have significantly more degrees of freedom than light field (LF) displays, resulting in higher resolution and better depth of field, but they do not natively support view-dependent effects and occlusions. Holographic stereograms (HS) and phase-added stereograms (PAS) utilize the capabilities of the holographic medium to increasing degrees, but also provide limited image quality. The proposed overlap-add stereograms (OLAS) provide the highest image quality among stereogram variants. Furthermore, the hogel size required by the OLAS is smaller than the Fresnel fringe pattern by a factor of the angular resolution of the hogel, reducing computation.

promising technologies for achieving many of these characteristics is holography.

Impressive results have recently been achieved by experimental holographic near-eye displays [Jang et al. 2018, 2017; Maimone et al. 2017], such as dynamic focus or eyebox expansion via eye tracking as well as vision and aberration correction. Yet, these systems rely on simple algorithms that use wavefront propagation to compute the hologram. Unfortunately, occlusions and view-dependent effects such as reflections are not natively supported by these computergenerated holography (CGH) algorithms. To overcome these limitations, holographic stereograms (HS) were proposed as CGH algorithms that directly encode a light field in a hologram [Kang et al. 2008; Yamaguchi et al. 1993; Yatagai 1976]. Light fields have the advantage that they contain occlusions and complex light transport effects, they can be rendered using computer graphics techniques, and emerging light field cameras can even capture cinematic content.

However, existing HS CGH algorithms struggle with creating a complex wavefront that successfully reproduces the information contained within the light field. A major source of the difficulty in recreating the light field is the tradeoff that comes from choosing the hogel size. Typically, an HS improves angular resolution at the expense of spatial resolution as the hogel size increases. However, analysis of the observable light field produced by a hologram [Zhang and Levoy 2009] leads to the insight that traditional HS algorithms discard important information required in the reconstruction of the wavefront from the light field. We propose the overlap-add stereogram (OLAS) as a method that inverts the observable light field transform more accurately than previous stereogram algorithms to create a hologram from a light field. This is achieved by treating a stereogram as an inverse short-time Fourier transform (STFT) and allowing hogel overlap. A comparison of traditional algorithms and the OLAS can be found in Table 1.

Therefore, in this work, we

- propose a new light field-based holographic stereogram algorithm by introducing the OLAS method;

- demonstrate that the OLAS method is not bound by the spatioangular tradeoff as a function of hogel size, representing a significant break from previous stereogram algorithms;
- experimentally validate our algorithms with a custom holographic near-eye display prototype, and introduce a new variant of phase compensation for high-quality encoding on the SLM.

A discussion of the limitations of our work can be found in Section 6.1.

\section{RELATED WORK}

Our approach builds on a large and diverse body of related work. In the following, we outline the most relevant display technologies and computer-generated holography (CGH) algorithms.

\subsection{Holographic Displays}

Holography relies on wave optics effects, i.e. diffraction and interference, to synthesize three-dimensional images using coherent light. Recording and displaying holograms became possible with the invention of the laser in the 1960s and much research and development has focused on maturing optical holographic imaging techniques in the following decades [Benton and Bove 2008]. Dynamic holographic displays [Slinger et al. 2005; Yaras et al. 2010] typically use spatial light modulators (SLMs), such as phase-only liquid crystal on silicon (LCoS) devices [Zhang et al. 2014], together with computer-generated holography (CGH) algorithms.

\subsection{Computer-generated Holography}

Point-based wavefront propagation is one of the most popular methods for computing a hologram; we refer to this approach as Fresnel holography. Given a 3D point cloud, an RGB-D image, a triangle mesh, or a few layers of intensity information, each scene point can be treated as the center of a spherical wave, which is propagated in free space to the SLM plane where all the spherical waves of the scene are added and computationally interfered with the reference beam [Goodman 2005; Park 2017]. The resulting wave field on the SLM plane, i.e. the hologram, contains phase and amplitude information. Unfortunately, most SLMs can only module either phase or amplitude but not both simultaneously. To approximate the complex-valued target field with phase-only information, two classes of algorithms have been proposed: iterative phase-retrievaltype methods [Dorsch et al. 1994; Fienup 1978, 1982; Gerchberg 1972; Jaganathan et al. 2015; Peng et al. 2017] and direct methods. Iterative methods minimize the reprojection error of the target intensity distribution using iterative optimization and are therefore usually slow. Direct methods are much faster and represent the target field using some form of double-phase [Hsueh and Sawchuk 1978; Maimone et al. 2017] or detour-phase [Burckhardt 1970; Lee 1970] coding. Naïve wavefront propagation methods can lead to ghosting effects, but more sophisticated methods also consider occlusions between objects in the propagation model [Chen and Wilkinson 2009; Matsushima and Nakahara 2009; Wakunami et al. 2013; Zhang et al. 2017, 2011] or simple non-Lambertian reflectance functions [Park 2017]. In general, view-dependent effects such as reflections are not natively supported by wavefront propagation methods. Moreover, this approach is limited in that it cannot easily deal with multiview photographs as the input for the algorithm. In contrast, we propose a light-field-based CGH algorithm that natively encodes 
complex view-dependent effects. It should be noted that there are several methods of calculating holograms [Park 2017] (e.g., angular spectrum methods) that we leave out for brevity.

\subsection{Holographic Stereograms}

Holograms that encode light fields are known as holographic stereograms (HS). The basic primitive of an HS is a holographic element, or hogel, which can be thought of as a small sub-hologram [Lucente 1994]. The first HS was optically recorded in 1967 by sequentially exposing the views of a light field into a holographic emulsion [Benton 1983]. Proposals on computer-generated stereograms followed shortly after [Yatagai 1976] and real-time implementations of these were later demonstrated [Lucente and Galyean 1995], made possible by HS algorithms only having $O\left(m^{2} \log n\right)$ complexity, for an $n \times n$ hogel size and $m \times m$ hologram resolution. The primary challenge of an HS is that hogels are treated as being independent and they are thus out of phase with one another, leading to low resolution and limited depth of field compared with Fresnel holograms. To remedy this, phase-added stereograms (PAS) introduce an extra phase factor to the hogel calculation to keep them in phase across the hologram [Yamaguchi et al. 1993]. Additional improvements of PAS algorithms, such as the accurate PAS (APAS), compensated PAS (CPAS), and the fast PAS (FPAS) have been proposed over the last few years [Kang et al. 2016, 2008], all of which are focused on further improving either computational complexity or quality of the wavefront approximation within the hogels. Most of these different PAS variants require a choice of hogel size that imposes a hard tradeoff: a larger hogel size to increase angular resolution and therefore depth of field, or a smaller hogel size to increase spatial resolution. The APAS is slightly different in this regard in that it computes a larger hogel for increased angular resolution, then crops hogels when tiling to achieve better spatial resolution (we will refer to these as the "input" and "output" hogel sizes, respectively). However, this cropping itself loses valuable information that our OLAS method takes advantage of to achieve better results in practice.

A very closely related method is found in concurrent work from Park and Askari [2019], who derive a similar algorithm via the Wigner transform. Their algorithmic complexity is similar to ours, roughly $O\left(m^{2} n^{2} \log n\right)$, but their algorithm does not use depth to align phases across hogels and instead relies solely on the information contained within the light field (e.g., as we must in Figure 9 for the scene reflected within the mirror). This lack of phase alignment leads to lower resolution in a way similar to how the phase alignment of the PAS improves the original HS algorithm. Their choice of a random phase carrier wave in their final results is similar to our randomized phase compensation (see Supplemental Figure S18), whereas we use Equation 4 to ensure a smooth phase profile.

It should be noted that $\mathrm{CGH}$ algorithms exist that make use of hogels, but that are not holographic stereograms. The approach taken by these methods is to process the light field of the scene using the Fresnel or other wavefront propagation method, but within each hogel instead of globally [Shi et al. 2017; Zhang et al. 2015]. This requires more computation than a typical HS, which only requires a single FFT per hogel, but much less than a full image wavefront propagation due to the relative smaller size of the hogels. Furthermore, since wavefront propagation is more accurate in the near field than an FFT, these algorithms also tend to produce better results. However, these and other algorithms that rely on Fresnel holography require different propagation kernels for different depths. This results in a different calculation per pixel, which may make parallelization more difficult, whereas the OLAS uses the same FFT and overlap-add independent of depth, possibly enabling dedicated accelerated hardware chips for these operations. Specifically, comparing the OLAS to Shi et al. [2017], we compute $n^{2}$ times more hogels, but each hogel only requires $n^{2} \log n$ for the Fourier transform. On the other hand, each hogel of Shi et al. requires roughly $n^{4}$ operations, though it may be more depending on the size of their kernel for wavefront propagation at each pixel, making the final complexity comparable. Moreover, the use of non-overlapping hogels introduces parallax error on the order of the hogel size in their method, which we avoid.

Lastly, there also exist stereogram algorithms that attempt to move beyond hogels to more complex primitives to increase computational efficiency and quality. For example Smithwick et al. [2010] proposed "wafels" and "direls" that specifically encode wavefront and directional information.

\subsection{Overlapping Hogels in Holographic Printing}

A method that at first glance seems to be related to the OLAS is that proposed by Hong et al. [2013] for holographic printing. However, there are two main differences. First, Hong et al. use a $50 \%$ shift between their hogels (i.e., adjacent hogel centers are spaced apart by $50 \%$ of the hogel size). While this is not necessarily incorrect to "invert" the hologram-to-light-field STFT (see Section 3), a 50\% shift requires stronger assumptions on some underlying window function for each hogel than shifting by a single pixel. The second difference makes clear that the OLAS is fundamentally different: overlap-add of hogels in the OLAS add coherently in the complex domain, whereas printed hogels (as in Hong et al.) are explicitly multiplexed such that they do not interfere with one another. This coherent addition is required to take full advantage of the degrees of freedom within a hologram and achieve maximum image quality.

\subsection{Holographic Near-eye Displays}

To diffract light in the visible spectrum efficiently, holographic displays require feature sizes on the order of a wavelength. This has the effect that SLMs used in holographic display are very small; this is particularly well-suited to near-eye applications which typically use magnifying optics. Several holographic near-eye display systems have been proposed, building on phase-only LCoS technology [Chen and Chu 2015; Li et al. 2016; Maimone et al. 2017; Moon et al. 2014; Yeom et al. 2015] or on multiple phase-only and/or amplitude-only SLMs [Gao et al. 2016; Shi et al. 2017]. Several recent systems also use eye tracking to overcome challenges associated with the limited space-bandwidth product of available SLMs, such as limited axial resolution or eye box size [Jang et al. 2018, 2017; Maimone et al. 2017]. These methods differ mostly in systems-level aspects, such as the type of SLM or optical combiner being used or whether eye tracking is used; almost all of them rely on the same 
two classes of CGH algorithms: point- or layer-based wavefront propagation. For example, Maimone et al. [2017] use the Fresnel method, which we compare against in this work; however, note that for the efficient implementation, Maimone et al. instead use a single-plane approximation to the Fresnel method, which takes only $O\left(m^{2} \log m\right)$ operations as opposed to roughly $O\left(\mathrm{~m}^{4}\right)$ of the true depth-varying method. The primary contributions of our paper are focused on novel algorithmic approaches to computer-generated holography (CGH), although we demonstrate them experimentally with a full-color holographic near-eye display.

\subsection{Near-eye Light Field Displays}

Much like holograms describe a scene using a wave field with spatially varying phase and amplitude, light fields describe the same concept using ray optics models. Building on these models, near-eye light field displays have been proposed for both VR [Huang et al. 2015; Lanman and Luebke 2013] and AR [Hua and Javidi 2014; Jang et al. 2017] applications. Although most of these displays have the advantage of using incoherent light, which is in line with most commercial microdisplays, they all suffer from limitations that prevent their adoption. Microlens-based light field displays [Hua and Javidi 2014; Lanman and Luebke 2013] make a fixed spatio-angular resolution tradeoff that is defined by the pixel and microlens sizes, limiting image resolution and achievable depth of field [Zwicker et al. 2006]. Multilayer liquid crystal displays (LCDs) [Huang et al. 2015; Lanman et al. 2010, 2011; Wetzstein et al. 2011, 2012] overcome this fixed tradeoff using content-adaptive light field factorization algorithms. However, the light efficiency of these displays is limited by the employed LCD technology and they require multiple LCDs, which increases cost and calibration complexity. Scanned laser displays [Jang et al. 2018, 2017] sequentially draw each ray or each view of a light field and their resolution is thus limited by the speed of the scanning mechanism or the spatial light modulator (SLM). Others have taken advantage of focus-tunable lenses and the fast refresh rates of digital micromirror devices (DMDs) to selectively light up volumetric pixels [Chang et al. 2018; Lee et al. 2019; Rathinavel et al. 2018], but must trade off framerate and depth resolution. In contrast to these technologies, holographic near-eye displays only require a single SLM with relatively low refresh rates and they offer significantly more degrees of freedom than incoherent light field displays, optimizing image resolution and depth of field.

\section{STEREOGRAMS AND THE LIGHT FIELD}

In this section, we review the role of the short-time Fourier transform (STFT) in connecting the hologram to the light field, and how holographic stereograms and phase-added stereograms attempt to invert the STFT.

\subsection{The Observable Light Field of a Hologram}

The wave optics analog of the light field is the Wigner distribution function (WDF) [Bastiaans 1978; Zhang and Levoy 2009]. Much like a spectrogram for one-dimensional signals such as sound, the WDF is a joint representation of space and spatial frequency (or angle). Unlike a light field or spectrogram, however, the WDF contains negative values to model interference and diffraction. Zhang and

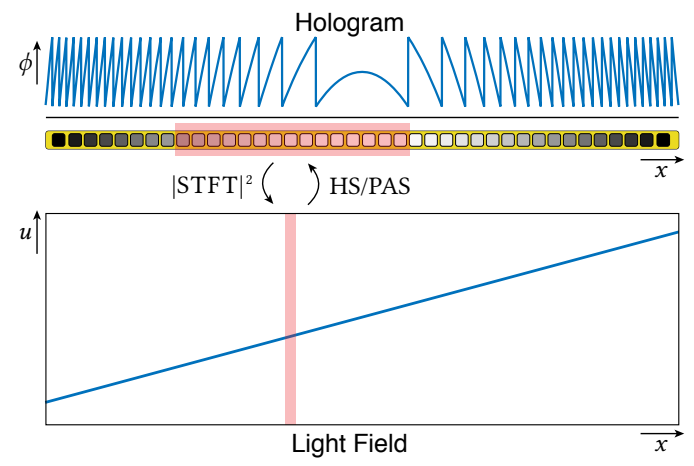

Fig. 2. Illustration of hologram-to-light-field transform. This example shows a single point at some distance to a phase-only hologram; the ground truth phase hologram is a Fresnel zone (blue, top) and the light field is a sheared line (blue, bottom). A hologram can be converted to a light field using the squared magnitude of the short-time Fourier transform (STFT). In this process, phase information is lost. Holographic stereograms aim to invert this ill-posed problem to compute a hologram from a light field by either ignoring the missing phase information (e.g., HS) or by approximating the phase using additional depth information (e.g., PAS).

Levoy [2009] showed that the observable light field is a local average of the WDF that satisfies the uncertainty principle. Though some have tried to directly use the WDF to synthesize a physically correct hologram from a light field [Hamann et al. 2018], this approach was only demonstrated with a handful of hogels as it requires solving an optimization problem per hogel and is in general computationally infeasible.

The short-time Fourier transform (STFT) provides a computationally efficient tool for computing the observable light field directly from a hologram [Zhang and Levoy 2009; Ziegler et al. 2007]. As illustrated in Figure 2, a sliding window $w$ of finite size is used to compute local Fourier transforms of the complex-valued hologram $h$; the angular distributions of the observable light field $L$ at the window centers are given by the squared magnitude of these Fourier transforms as

$$
\begin{aligned}
& L\left(x, y, u_{x}, u_{y}\right)=\left|\operatorname{STFT}\left\{h\left(x^{\prime}, y^{\prime}\right)\right\}\left(x, y, u_{x}, u_{y}\right)\right|^{2} \\
& \quad=\left|\int_{-\infty}^{\infty} h\left(x^{\prime}, y^{\prime}\right) w\left(x^{\prime}-x, y^{\prime}-y\right) e^{-i\left(u_{x} x^{\prime}+u_{y} y^{\prime}\right)} d x^{\prime} d y^{\prime}\right|^{2},
\end{aligned}
$$

where $w$ is a window function; we use a Hann window. Here, we parameterize the light field $L$ using spatial coordinates $x, y$ and spatial frequencies $u_{x, y}=\sin \left(\theta_{x, y}\right) / \lambda$, which are directly related to the corresponding angles $\theta_{x}$ and $\theta_{y}$.

What is crucial to understand is that the STFT of a hologram contains both amplitude and phase, and is thus invertible under certain conditions, while its squared magnitude, i.e. the observable light field or spectrogram, does not contain phase information and is therefore not invertible. In essence, all variants of holographic stereograms amount to solving the ill-posed problem of inverting a spectrogram. 


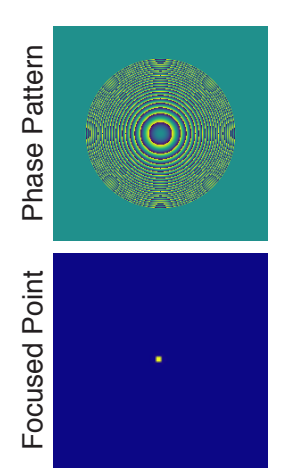

Fresnel

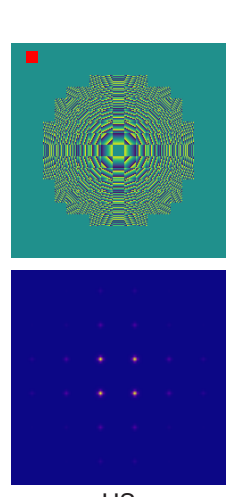

HS
Hogel Size $13 \times 13$
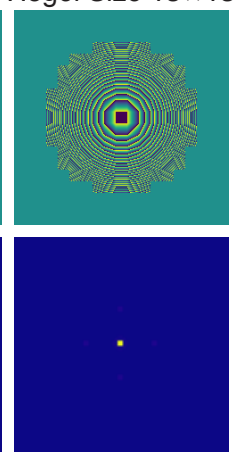

PAS/APAS

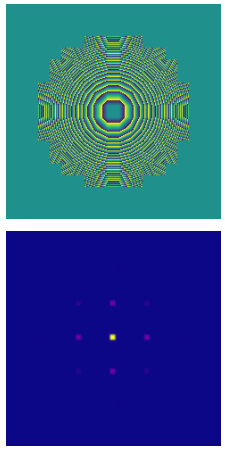

OLAS

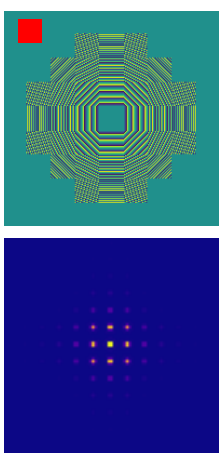

HS

Hogel Size $26 \times 26$

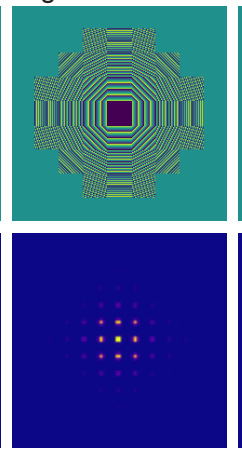

PAS/APAS

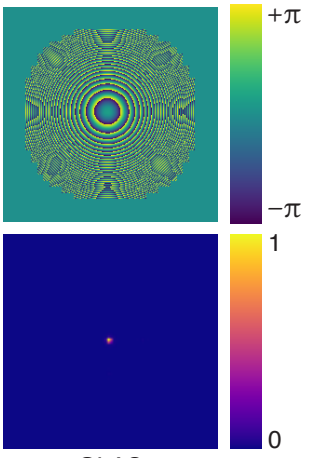

OLAS

Fig. 3. Phase profiles (top row) of a point $1.3 \mathrm{~cm}$ from the hologram plane and the corresponding focused spot at the target distance (bottom row). A Fresnel hologram provides the reference phase profile. Holographic stereograms (HS) approximate the phase profile with a discrete set of hogels. This approach has poor focusing capabilities because the hogels are out of phase. Phase-added stereograms (PAS) use depth information of the scene to calculate hogels that are in phase, often improving the quality of the hologram. However, as the hogel sizes increase beyond an optimum, the focused point develops aliasing artifacts. On the other hand, the proposed overlap-add stereogram (OLAS) provides a high resolution reconstruction of the focused point independent of hogel size. Note that the distance to the point has been specifically selected to coincide with the discretized angular sampling of the PAS, making it equivalent to an accurate PAS (APAS). Also note that the Fresnel spot appears larger than the $26 \times 26$ OLAS spot only because it is centered at a corner of the pixel grid, whereas the OLAS is not due to a sampling location implementation detail that shifted the output point by $1 / 4$ of a pixel. This does not affect more complex images. The hogel size is indicated by the red square for scale.

\subsection{Holographic Stereograms (HS)}

As outlined in Section 1, the most basic light field-based CGH algorithm is the holographic stereogram [Yatagai 1976]. The idea is intuitive: given the angular variation of a light field $L\left(\theta_{x}, \theta_{y}\right)$ at a fixed position $x_{0}, y_{0}$, the hologram creates a set of plane waves that locally approximate $L$. This idea is closely related to microlensbased light field displays, where angle-dependent radiance values at some position are optically encoded by interleaving them behind a microlens. In an HS, the same information is optically encoded using a diffractive fringe pattern within a hogel. In HSs, hogels are treated as if they were mutually incoherent: i.e., while diffraction creates the plane waves within a hogel, light from different hogels is treated as either not interfering or interfering only in a random manner. Therefore, spatio-angular resolution tradeoffs of HS and microlens-based light field displays are equivalent and overall poor compared to other types of holograms [Zwicker et al. 2006].

To compute a hogel from the light field angles at $\left(x_{0}, y_{0}\right)$, we rely on a key insight from Fourier optics: a plane wave propagating at angle $\theta$ corresponds to the spatial frequency $u=\sin (\theta) / \lambda$ [Goodman $2014]$. Thus, the $2 \mathrm{D}$ Fourier transform of $L\left(\theta_{x}, \theta_{y}\right)$ directly computes the coefficients of each plane wave. This is shown in Figure 3 for the example of a single point $1.3 \mathrm{~cm}$ from the hologram. The reference phase in the top left is computed by propagating the point to the hologram plane using the Rayleigh-Sommerfeld diffraction integral. The holographic stereogram discretizes the hologram, here shown for hogels with a resolution of $13 \times 13$ and $26 \times 26$, and approximates the wavefront of the point locally with a single plane wave, i.e. a small grating with a specific spatial frequency and orientation, that optically steers light toward the point. The computed hogels are mutually incoherent, or out of phase, and the "focused" point created by this hologram (Figure 3, bottom row) is much bigger than that of the Fresnel hologram. The effective numerical aperture of the latter corresponds to the size of the entire hologram whereas that of the HS is only the size of the hogel. Pseudocode for the HS algorithm and additional simulations for varying hogel sizes are included in the supplement.

\subsection{Phase-added Stereograms (PAS)}

Holographic stereograms aim to invert Equation 1 by simply ignoring the missing phase information of the STFT. After all, a light field has no phase. However, Yamaguchi et al. [1993] realized that depth can be used to compute the missing phase. Specifically, if not only the light field $L$ is available but also a "depth field" $D$ that contains depth information for each ray, then the STFT can be calculated from the spectrogram (i.e., the light field $L)$ as STFT $=\sqrt{L} \exp \left(i \frac{2 \pi}{\lambda} D\right)$. Given both amplitude and phase, the procedure outlined in the previous subsection can be followed to calculate a phase-added stereogram from the STFT in a hogel-by-hogel manner. As seen in Figure 3 for the $13 \times 13$ hogel size, this brings individual hogels "in phase" and allows for a significantly brighter focused spot because all hogels interfere with one another.

The accurate phase-added stereogram (APAS) method [Kang et al. 2008] improves upon the PAS by computing the local Fourier transforms on an input light field with higher angular resolution, providing a slightly better quality than the HS and PAS. Specifically, the higher angular resolution hogel is Fourier transformed, and then cropped down to the target "output" hogel size. This cropping before tiling allows the APAS to maintain the spatial resolution of a much smaller PAS while benefiting from finer angular resolution from the larger "input" hogel size. Although other improved variants of the APAS have been discussed in recent work [Kang et al. 2016], these 

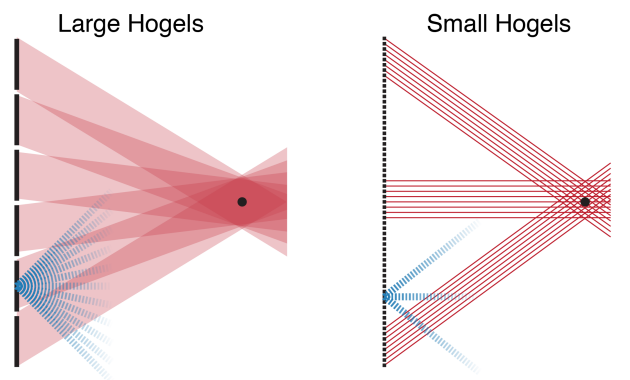

Fig. 4. The effect of hogel size on spatial and angular resolution when trying to reconstruct a point. Holographic and phase-added stereograms largely treat the hogels independently when calculating the correct pattern to display. Each hogel (black rectangles) contributes a single plane wave to the reconstruction, approximating a spherical wavefront. The number of pixels in a hogel determines the spatial extent of the plane wave, but also the number of non-overlapping spatial frequencies, or directions, that can be encoded for that plane wave. The former leads to larger hogels exhibiting worse spatial resolution (left). The latter leads to smaller hogels having worse angular localization (right). A hogel with 3 pixels for example can only represent 3 non-overlapping spatial frequencies or angles (we show the central ray directions for a single hogel in blue). While the Fourier transform limits the HS and PAS to evenly spaced angles, other methods can provide finer control over the central ray direction; however, the larger angular spread of smaller hogels still imposes the same limit on the number of resolvable angles. Typically, closer points benefit from smaller hogels and farther points from larger hogels.

methods require knowledge of a point's location prior to quantization on a pixel grid, such as by using point clouds as input to the $\mathrm{CGH}$ algorithm. On the other hand, the APAS and our approach rely exclusively on light field and depth information. Pseudocode for the PAS and APAS as well as additional simulations are included in the supplement.

\subsection{Hogel Size and the Resolution Tradeoff}

One important question for all holographic stereogram algorithms is how to choose the hogel size. Since HS algorithms use nonoverlapping tiles, larger hogels decrease the spatial resolution that can be displayed (Figure 4). On the other hand, larger hogels improve the angular resolution of an HS, i.e. the accuracy of orienting plane waves created locally within a hogel. Higher angular resolution, in turn, increases the depth of field, i.e. the resolution of an object at some distance $z$ from the hologram plane. In effect, stereograms exhibit the same spatio-angular resolution tradeoff as microlens-based light field displays.

In effect, this means the optimal hogel size is heavily influenced by the depth of the object to be encoded, which makes the choice of hogel size for a stereogram extremely scene dependent (see the supplement for how the window length of the STFT, depth, and hogel size are related). This scene dependence makes hogel size a difficult parameter to tune and also makes complex scenes difficult to display. Breaking the depth-dependence of hogel size and resolution is crucial to enabling the construction of complex scenes with holography.
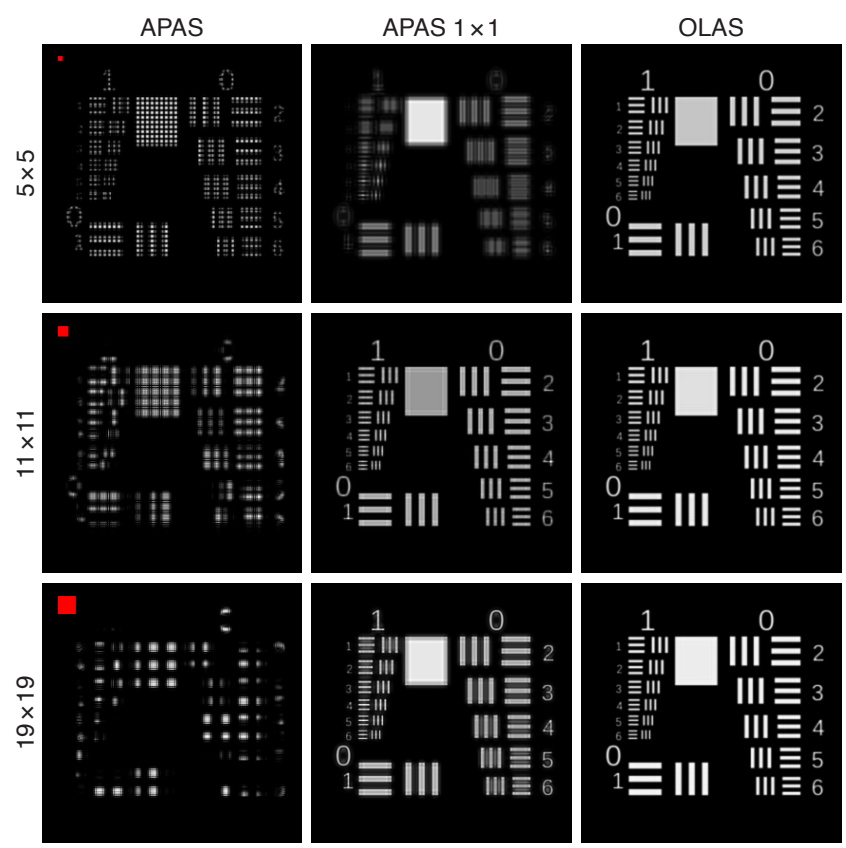

Fig. 5. Comparison of the APAS and OLAS algorithms and hogel sizes for a simulated resolution chart at a small distance to the hologram plane. The hogel size is indicated to the left of each row, and the red square in the top left corner shows the hogel relative to the image for scale. For the APAS, the indicated hogel size corresponds to the output hogel size after cropping an input hogel that is $3 \times$ larger. This shows the effect of decreasing spatial resolution with hogel size. The "APAS $1 \times 1$ " uses the indicated hogel size as the input size, with the output set to $1 \times 1$. It can be seen that despite the constant output hogel size, the $1 \times 1$ APAS still requires a distancedependent optimal input hogel size: in this case, $11 \times 11$. Smaller hogel sizes are blurry and larger sizes exhibit haloing and other edge artifacts. The OLAS algorithm provides the best performance at all hogel sizes by making full use of the angular information when inverting the STFT. The overlapping hogels do not noticeably degrade resolution with larger hogels.

\section{OVERLAP-ADD HOLOGRAPHIC STEREOGRAMS}

We propose a new variant of the phase-added stereogram method, dubbed the overlap-add stereogram (OLAS). This algorithm is based on the overlap-add method to invert the short-term Fourier transform [Allen 1977]. As opposed to other variants of the HS or PAS, the OLAS uses a sliding window. At each spatial location of the input light field, the angular radiance distribution and the depth information are converted, via a Fourier transform, into a set of local plane waves. The resulting complex-valued hogel is multiplied by a window function $w$, such as a Hann window, and added to the hologram as

$$
\begin{aligned}
& h(x, y)=\operatorname{STFT}^{-1}\left\{\sqrt{L\left(x, y, u_{x}, u_{y}\right)} e^{i \frac{2 \pi}{\lambda} D\left(x, y, u_{x}, u_{y}\right)}\right\} \\
& =\int_{-\infty}^{\infty} \int_{-\infty}^{\infty} w\left(x-x^{\prime}, y-y^{\prime}\right) \iint_{-\infty}^{\infty} L\left(x^{\prime}, y^{\prime}, u_{x}, u_{y}\right) \\
& e^{i 2 \pi\left(u_{x} x^{\prime}+u_{y} y^{\prime}+\frac{D\left(x^{\prime}, y^{\prime}, u_{x}, u_{y}\right)}{\lambda}\right)} d u_{x} d u_{y} d x^{\prime} d y^{\prime} .
\end{aligned}
$$


Like the PAS, the OLAS requires dense light field depth information $D\left(x, y, u_{x}, u_{y}\right)$. Pseudocode for the OLAS method is outlined by Algorithm 1. Here, $s, t$ are the local hogel coordinates and $m, n$ are hogel width and height. The use of $D$ to compute $\phi$ ensures that the overlapping hogels are in phase and constructively interfere, thereby improving the angular resolution and ability to represent view-dependent effects as the light field density increases.

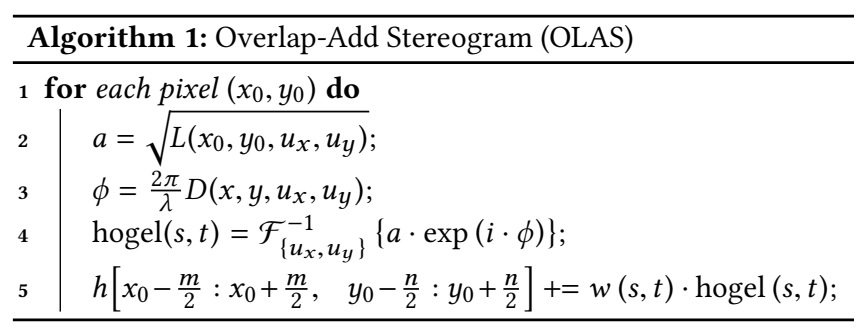

A comparison of the OLAS, Fresnel holograms and other holographic stereograms is shown for a single point target in Figure 3 . While for some hogel sizes, the PAS and APAS may produce a well-defined point, we note that the OLAS produces the best approximation of the reference Fresnel phase profile without regard to the hogel size used. It can also be seen in the figure that the shape of the fringe pattern produced by the OLAS approaches that of the Fresnel pattern as the hogel size, and therefore angular sampling density, increases. There are some remaining artifacts in the shape of the point due to the shape of the overlap-add region not matching an ideal circular fringe pattern, but this does not affect the overall quality of other reconstructed objects (e.g., Figure 5).

\subsection{Hogel Size-Independent Resolution}

The OLAS method breaks the traditional tradeoff between spatial and angular resolution by allowing large hogels to overlap and shift by only a single pixel at a time. Often when faced with a resolution tradeoff, sampling a field more densely serves to only spuriously increase pixel resolution without changing the underlying real resolution, e.g., when using a camera sensor with pixels smaller than the diffraction limited resolution of the optics. Surprisingly, the OLAS breaks this trend, and the interference in the complex domain of adjacent hogels successfully recovers greater spatial information without sacrificing the angular resolution. As evidenced by Figure 5, we can freely increase the hogel size to much larger than the smallest feature size without degrading image quality.

While at first glance, the OLAS may seem to offer no advantage over using an APAS with an output hogel size of $1 \times 1$, the cropping operation loses information that would normally be required to invert the STFT correctly. We see in the simulated results (Figure 5 , middle column) that the APAS has artifacts near edges and still exhibits a dependence on hogel size, with some optimum that depends on depth. Finally, the APAS is less "efficient" than the OLAS due to the loss of information and requires a larger input hogel size to achieve reasonable resolution.

Importantly, the invariance of the reconstructed image quality to hogel size points to another advantage of the OLAS method over not only other stereogram methods, but also over Fresnel methods. Recall from Section 3.4 that the optimal hogel size for stereograms is tied to the depth of the object being reconstructed. Using Fresnel zones to propagate individual points also requires a different sized fringe pattern for different depths - specifically, a pattern with radius $r=z \tan \left(\theta_{\max }\right) / p$ that depends on the maximum diffraction angle, $\theta_{\max }$, and the pixel pitch of the SLM, $p$. Larger Fresnel fringe patterns will alias as the spatial frequency exceeds that supported by the pixel pitch. The OLAS method, by producing high resolution reconstructions independent of hogel size - a parameter typically tied to depth - is therefore also independent of object depth and is scene invariant.

It should be noted that while the OLAS does not seem to suffer negative effects from arbitrarily large hogel sizes, the algorithm is not designed to hallucinate information not otherwise present in the light field. This means that if the underlying light field is too sparsely sampled angularly, the OLAS must also necessarily exhibit reconstruction artifacts (see supplement for simulations). Specifically, the light field is undersampled for our purposes when the number of pixels a given point shifts spatially between angular views of the light field exceeds the size of the hogel (e.g., Figure S6). The amount of shift between angular views is equal to the size of the diffraction cone at that distance divided by the total number of angular samples. Since the number of angular samples is exactly equal to the hogel size, we can calculate that the maximum amount of inter-view shift-and therefore the effective minimum hogel size - as $\sqrt{z_{\max } \tan \left(\theta_{\max }\right) / p}$. Though this minimum hogel size is imposed by the farthest plane, the hogel size-invariance makes it a suitable hogel size for all closer distances as well. This is in contrast to other CGH algorithms where increased hogel size to accommodate farther points negatively affects closer points (for which typical HS algorithms would require a smaller hogel size, and Fresnel methods would require a smaller fringe pattern).

Additional simulations of the point targets and resolution charts at different depths and with different hogel sizes can be found in the supplement. Further comparisons of the various algorithms with these parameters are also present.

\section{IMPLEMENTATION AND ASSESSMENT}

\subsection{Prototype Near-eye Display}

Hardware. The spatial light modulator in our prototype is a HOLOEYE LETO (VIS-009) phase-only LCoS with a resolution of $1920 \times$ 1080 (1080p) and a pixel size of $6.4 \mu \mathrm{m}$. This SLM provides a refresh rate of $60 \mathrm{~Hz}$ (monochrome) with a bit depth of 8 bits and a diffraction efficiency of over $80 \%$. The laser is a FISBA RGBeam fiber-coupled module with three optically aligned laser diodes with wavelengths of 638,520 , and $450 \mathrm{~nm}$. Color images are captured as separate exposures for each of the three wavelengths and combined in post-processing. We use two different eyepieces in our system: first is a Nikon AF-S Nikkor $50 \mathrm{~mm} \mathrm{f/1.4} \mathrm{lens} \mathrm{for} \mathrm{the} \mathrm{parallax} \mathrm{and}$ reflection results, and second is a Meade Series 5000 HD-60 25mm 6-Element Eyepiece (1.25") with $f=25 \mathrm{~mm}$ for the other results. Other components include a polarizer (Thorlabs LPVISE100-A), a collimating lens (Thorlabs C40FC-A), and a beam splitter (Edmund Optics, $25 \times 25 \mathrm{~mm}$ 50R/50T, VIS), as indicated in Figure 6. With the $50 \mathrm{~mm}$ eyepiece, the near-eye display offers a diagonal field of 


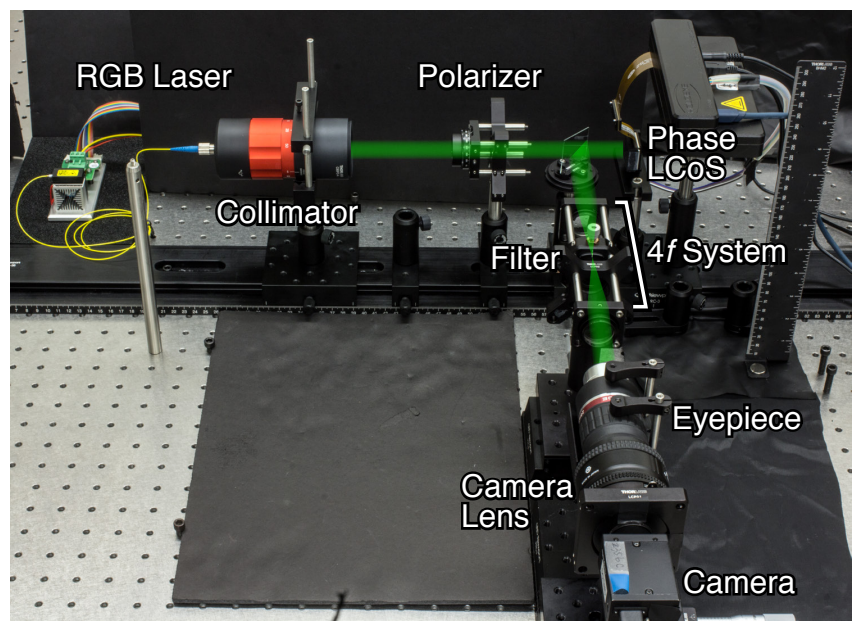

Fig. 6. A photograph of the prototype holographic near-eye display system showing the optical path. The beam is collimated and polarized before being modulated by the phase $\mathrm{LCoS}$, then enters a $4 f$ system with a variable aperture in the Fourier plane acting as a filter. Finally, it passes through the eyepiece and camera lens to be imaged by the camera. The RGB laser is hardware synchronized with the phase LCoS. The eyepiece is positioned $10 \mathrm{~cm}$ away from the image of the SLM. The camera lens is placed with its aperture at the eye box location.

view of $16.05^{\circ}$ and an eyebox of $4.07 \mathrm{~mm}$. With the $25 \mathrm{~mm}$ eyepiece, it offers a diagonal field of view of $31.5^{\circ}$ an the eyebox is $2.03 \mathrm{~mm}$. We set the zero-disparity plane of the light field to a virtual image distance of $d_{v}=66.7 \mathrm{~cm}$. A $4 f$ system $(f=50 \mathrm{~mm})$ is used to filter higher order diffraction artifacts, and images were captured with a Pointgrey Grasshopper3 2.3 MP Color USB3 Vision (Sony Pregius IMX174) imaged through a Nikon AF-S Nikkor 35mm f/2 lens.

Light Field and Hologram Rendering. Note that every location $v$ on the pupil plane directly maps to angle $\theta=\tan ^{-1}\left(v / d_{\text {eye }}\right)$ inside the HMD. This allows us to draw the link between the perspective projections of a target light field showing a scene from viewpoints $v$ and the spatial frequencies of the non-magnified hologram ${ }^{1}$. We rendered our light fields in Unity with a viewport placed at the virtual image distance $d_{v}=66.7 \mathrm{~cm}$, with near and far clipping planes set to $33.3 \mathrm{~cm}$ and $100 \mathrm{~m}$ (approximately a $3 \mathrm{D}$ range for depth of field). See Appendix for background on rendering light fields for holography. The viewport dimensions were $32.8 \mathrm{~cm} \times$ $18.4 \mathrm{~cm}$, corresponding to the magnified size of the SLM at the virtual image distance. Scenes were generated with $9 \times 9$ equally spaced views over the eyebox at $1080 \mathrm{p}$ resolution.

All calculations to convert the light field to a hologram via the OLAS method, and also those to implement the HS, PAS, and APAS baselines were done in MATLAB using RGB-D light fields output from Unity.

\footnotetext{
${ }^{1}$ In computer graphics, the light field is often represented using a two-plane parameterization on planes $x$ and $v$ [Levoy and Hanrahan 1996]. A target light field on the user side of the eyepiece can be rendered from a 3D model using perspective projections or photographed with a light field camera. The holography literature usually uses a parameterization based on space and spatial frequency. These spatial frequencies can be interpreted as orthographic projections on the SLM side of the eyepiece.
}

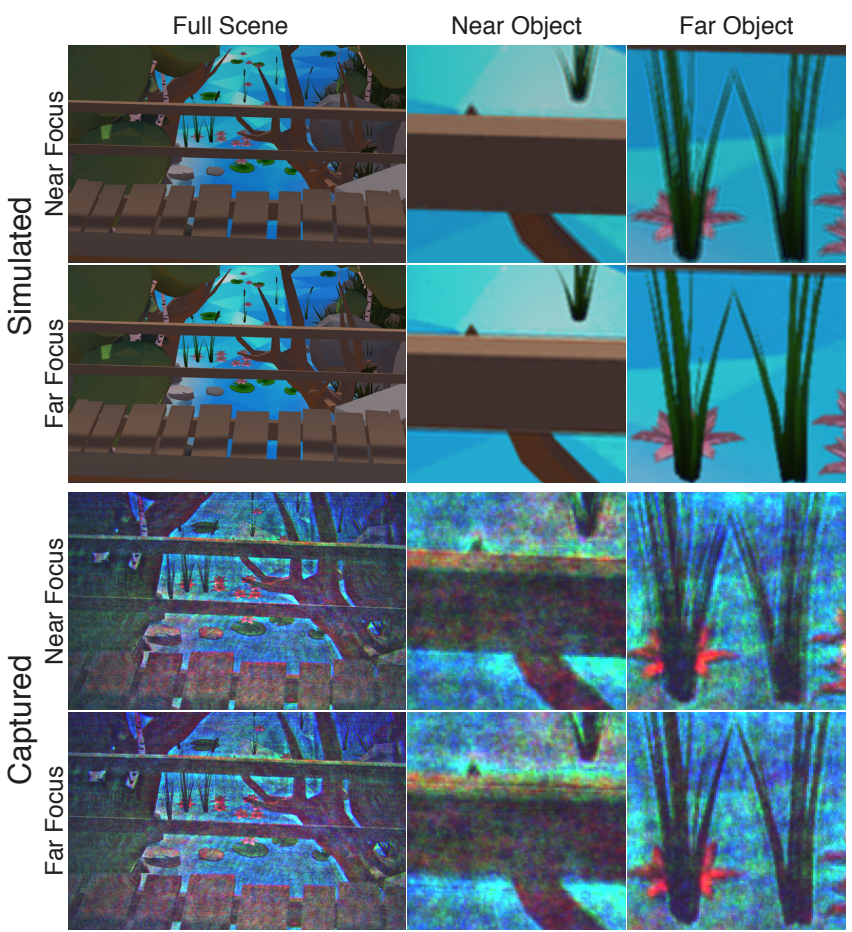

Fig. 7. Focal stacks for a selected scene at two different distances. The simulated results are generated by using Fresnel propagation from the output of the OLAS algorithm to the distance of the object of interest. It can be seen in the zoomed views to the right of the simulated and captured results that near and far objects come into focus at the correct near and far focus distances. Due to imaging through an eyepiece with $f=25 \mathrm{~mm}$, the supported range of 3 diopters corresponds to only $2 \mathrm{~mm}$ of optical path length, leading to only a small blur radius after defocus.

Displaying Stereograms with Phase-only SLMs. All stereogram variants compute a hologram $h$ that has both amplitude and phase. Simply discarding the amplitude to display $h$ directly on a phase-only SLM would be a very crude approximation of the desired information. We therefore propose to propagate $h$ by some distance $d_{1}$ (we set $d_{1}=10 \mathrm{~cm}$ ) in free space to the SLM plane, for example via the Rayleigh-Sommerfeld or Fresnel method [Goodman 2014], where it is optically encoded by the phase-only SLM (Figure 6). Iterative phase retrieval type algorithms (see Section 1) are not applicable in this case, because they only constrain the target intensity, i.e. $|h|^{2}$, whereas our approach constrains both amplitude and phase of $h$ at the hologram plane. To solve this problem, we use the double phase coding algorithm recently employed by Maimone et al. [2017]. This approach represents a complex field $c$ on the SLM plane with amplitude $a$ and phase $\phi$ as the sum of two phase-only fields,

$$
\begin{gathered}
\phi_{1}(x)=\phi(x)-\cos ^{-1} a(x) \\
\phi_{2}(x)=\phi(x)+\cos ^{-1} a(x) \\
c(x)=a(x) e^{i \phi(x)}=0.5\left(e^{i \phi_{1}(x)}+e^{i \phi_{2}(x)}\right) .
\end{gathered}
$$

It can be seen that $\phi_{1}(x)$ and $\phi_{2}(x)$ span a $3 \pi$ phase range, which means double phase coding requires an SLM that supports at least 


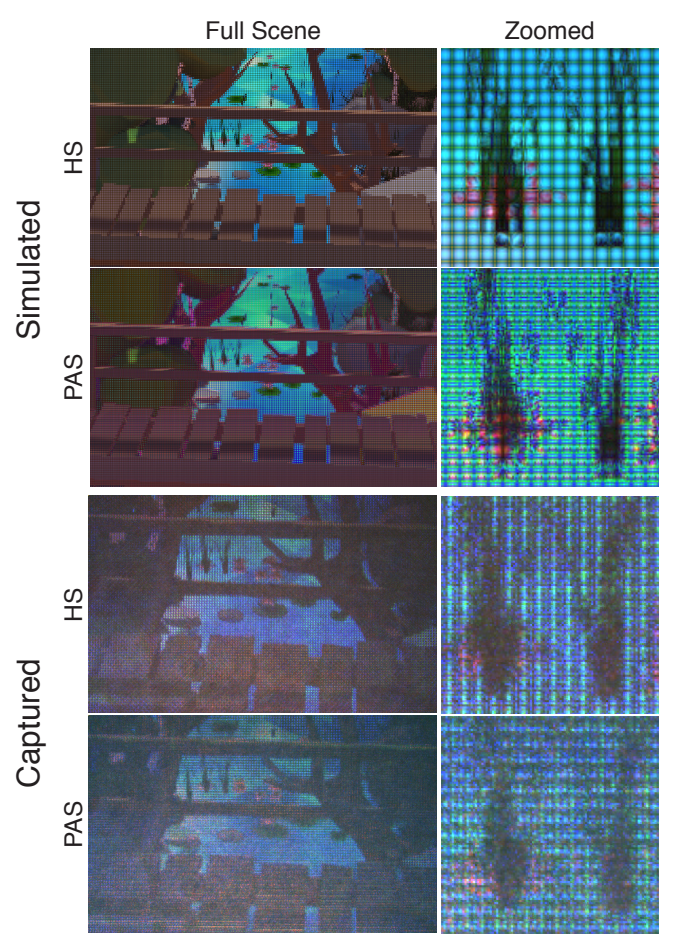

Fig. 8. Example simulation and capture of a scene using traditional stereogram algorithms such as the HS and PAS. The hogel size was set to $9 \times 9$, matching the OLAS. While the full scene looks fine, zooming in on a portion reveals the severely reduced spatial resolution.

$3 \pi$ phase modulation to avoid artifacts caused by phase wrapthis is not a theoretical requirement, but rather one to mitigate aberrations of current phase SLMs. While double phase coding is well known [Hsueh and Sawchuk 1978], Maimone et al. recently showed that the two phase-only fields can be interleaved into a single phase-only pattern if $\phi(x)$ is sufficiently smooth. When using the Fresnel method, a way to achieve a relatively smooth phase profile is to normalize the phase at the center of the Fresnel fringe pattern such that it is zero, regardless of the distance to the point This physically corresponds to assigning the correct initial phase to the point source [Maimone et al. 2017].

However, when dealing with a light field as the input, the exact correspondences between points across angular views is not known. Luckily, with a rendered light field, the distance from the camera plane to a pixel is easily obtained as part of the perspective projection. Given the camera positions of a given angular view, that "linear" distance can be transformed to the actual Euclidean distance, which we refer to as the "depth field" $D$. The final piece of information required comes from the Fourier transform applied to each hogel. The spatial frequency $u_{x, y}$ for each pixel within the hogel allows us to cast a ray in the approximate direction of the point quantized by the hogel resolution, $\hat{\theta}$, and with length given by $D$. Given this estimate of the point location, we can transform $D$ to a

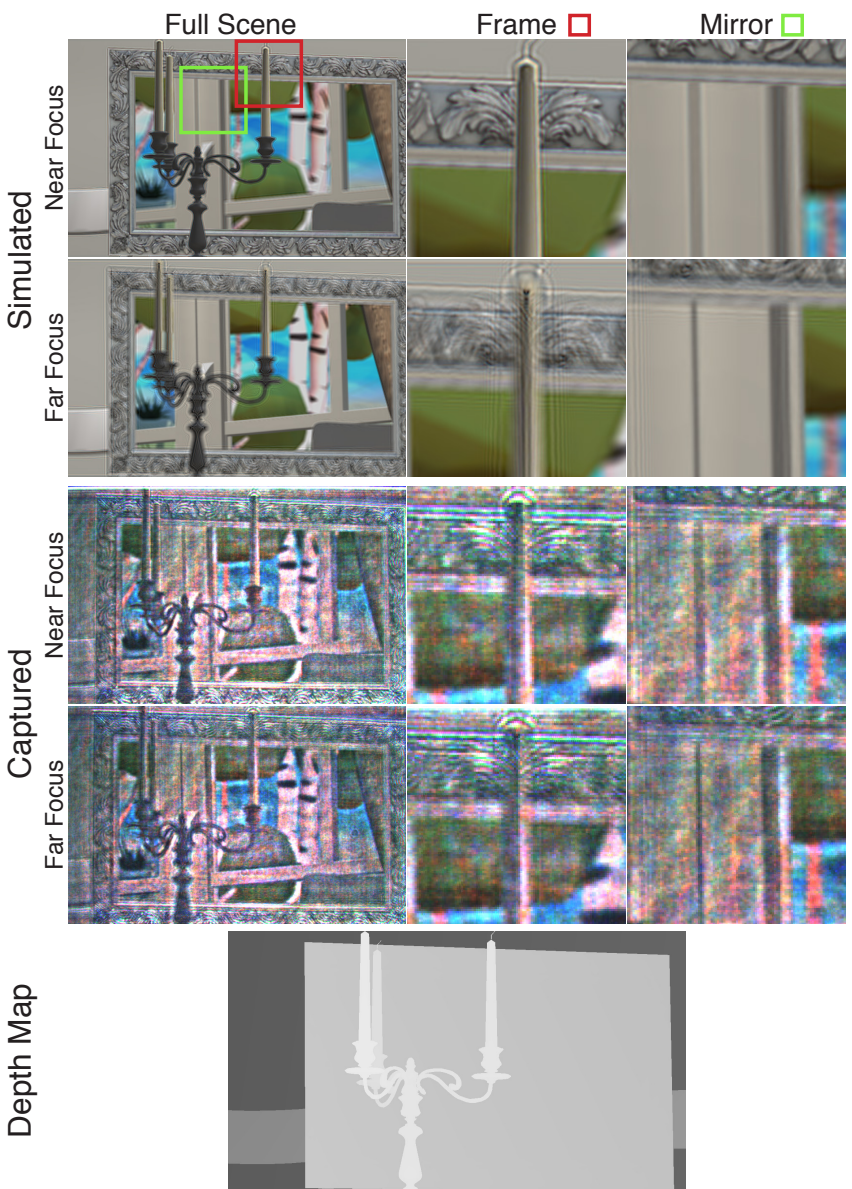

Fig. 9. Experimental results demonstrating a non-Lambertian surface. The depth map of the mirror is rendered as a flat plane, as expected. However, using information in the light field, holographic stereograms like the OLAS can recover some depth for the hologram. Note though that the lack of correct a depth map results in the objects within the mirror being out of phase, reducing resolution slightly. The slightly unnatural out-of-focus blur seen in the simulated images may be a ringing artifact caused by the limited spatial bandwidth of the propagation simulation.

normalized depth as

$$
\begin{aligned}
\hat{\theta}\left(u_{x}, u_{y}\right) & =\sin ^{-1}\left(\lambda \sqrt{u_{x}^{2}+u_{y}^{2}}\right) \\
D^{\prime}\left(x, y, u_{x}, u_{y}\right) & =D\left(x, y, u_{x}, u_{y}\right)\left(1-\cos \hat{\theta}\left(u_{x}, u_{y}\right)\right),
\end{aligned}
$$

where $D \cos \hat{\theta}$ gives the approximate distance of the point directly to the hologram plane.

Although the approach to displaying holographic stereograms with phase-only SLMs described in this subsection builds on a set of previously discussed techniques, we are not aware of prior work that combines them in the proposed manner.

In our implementation, we choose to use the depth compensation given by Equation 4 to ensure smooth phases for the double phase coding to achieve high image quality. The effect of other treatments of the phase, such as directly using the uncompensated $D$ or using 
a random phase to simulate diffuse objects, is discussed further in the supplement.

\subsection{Results}

The images generated on the SLM by the OLAS method can be found in Figure 7 alongside simulated results showing the theoretical quality of the algorithm. As evidenced in the figures, the OLAS method supports refocus to multiple depths. Unlike other stereogram algorithms (Figure 8), the OLAS also produces images with much higher spatial resolution. This is a direct result of overlapping instead of tiling hogels. Next, we generate a hologram containing a non-Lambertian surface, specifically a mirror (Figure 9). Despite the depth map given by the light field appearing as a flat plane, the simulated and captured results make clear that the mirror's frame and objects reflected within are in fact at different depths. Finally, in Figure 10, we look at the parallax and occlusions produced by the OLAS method. Compared to the same scene generated using the Fresnel method, it can be seen that the OLAS method produces more accurate occlusions and parallax (see supplemental video). This matches expectation since stereograms natively encode such effects.

\section{DISCUSSION}

Holographic stereograms are capable of encoding information from a light field into a hologram. They rely on the hogel as a building block for assembling a larger hologram. However, most HS implementations rely on tiling non-overlapping hogels, which impose the same spatio-angular tradeoff as microlens-based light field displays. In the case of microlenses, the lack of overlap is a physical constraint. On the other hand, holography relies heavily on superposition and linearity, making the lack of overlap between hogels an arbitrary choice.

Based on the insight that the observable light field of a hologram can be calculated as the squared magnitude of the hologram's STFT [Zhang and Levoy 2009], we analyze HS algorithms in the context of the STFT. Based on our analysis, we propose the OLAS as a method of creating a new type of HS which is no longer constrained to non-overlapping hogels. In the context of the STFT, the OLAS uses depth information to recover phase information not present in the light field, followed by the overlap-add method of inverting the STFT itself. By allowing overlap, the OLAS creates smooth variation across hogels and avoids edge artifacts necessarily present in tiled approaches. Furthermore, by not cropping as done by the APAS, the OLAS preserves more of the information of the target light field, allowing formation of higher resolution images at smaller hogel sizes.

Most importantly, the per-pixel shift enabled by overlap-add results in high spatial resolution images regardless of increasing hogel size. This allows the OLAS to take advantage of both high spatial and angular resolution, displaying depth- and hogel size-invariant performance on tested scenes. This is in contrast to not only HS algorithms, but also to Fresnel methods, which require different Fresnel zones for each depth.

\subsection{Limitations and Future Work}

While our simulations show clear imagery of the effects of our algorithm, the physical implementation leaves much to be desired. Several reasons conspire to produce reduced image quality in practice. In our testing, nonuniformity and residual nonlinearity of phase quantization levels seems to have the greatest effect. Across all wavelengths, the quantization of the phase range is much finer in the $0-2 \pi$ range than in the $2 \pi-3 \pi$ range (required for double phase coding). The nonuniform quantization degrades image quality (see supplement for simulations); to mitigate this, we recenter our phases such that the majority of phases lie within the $0-2 \pi$ range, but there are inevitably phases beyond this range after double phase coding. We expect that the image quality would be greatly improved with a different SLM. Double phase coding also adds another artifact in the form of ghost images caused by the high frequency checkerboard pattern of the coding pattern itself. These ghost images are formed closer than the typical higher diffraction orders, making them harder to filter out without also losing angular information in the hologram itself for view-dependent effects and depth of fieldwe necessarily had to leave some of these ghost images unfiltered to
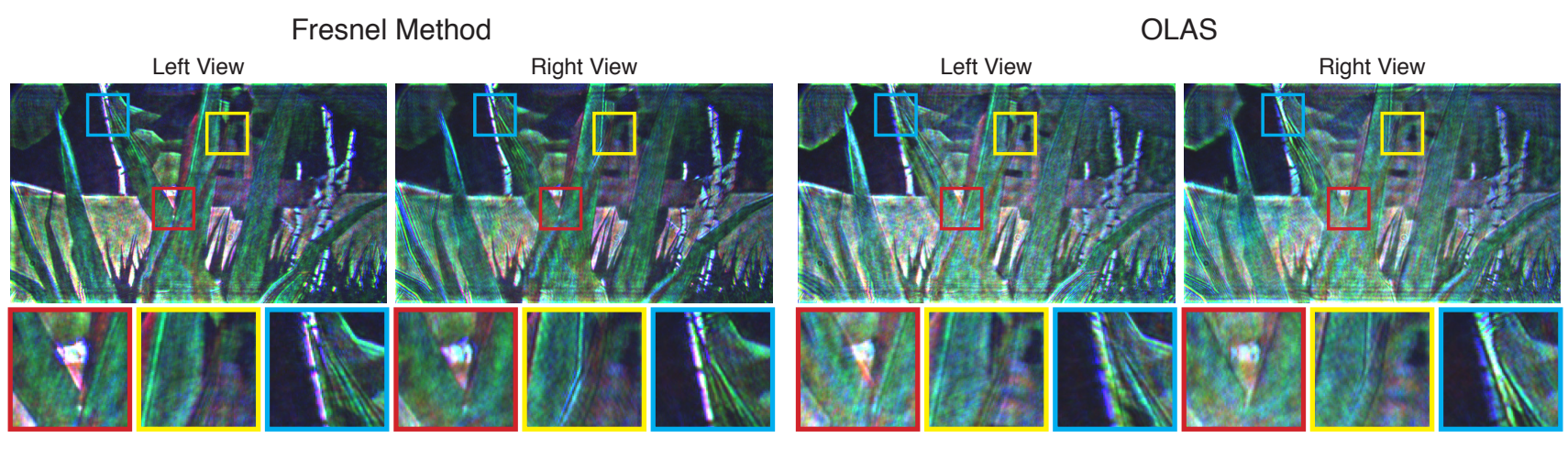

Fig. 10. Experimental results demonstrating parallax. We compare the Fresnel method against the OLAS. In general, evidence of parallax (with a 4 mm baseline) can be seen with both methods; however, the OLAS performs better. In the zoomed views along the bottom, it can be seen that (red) only the OLAS reveals the ground in the gap between the blades of grass in the right view, (yellow) the Fresnel method results in one of the blades of grass almost disappearing in the left view, and (blue) the grass actually moves relative to the white tree in the OLAS method. See supplemental video for animated comparison. 
be able to demonstrate our method. A possible method of mitigating both of these issue would be to add an amplitude modulator to the optical path, which would obviate the need for double phase coding and a $3 \pi$ phase range.

There are various other limitations of our current SLM setup that may affect the image quality. One is drift in the phases due to temperature variance over time, which affects both the quality of our phase lookup table (LUT) and also the accuracy of any LUT for the current state of the system. Another is the lack of filtering of the zero order, which reduces image contrast to an extent. We also notice some optical aberrations caused by our lenses, notably some interreflections between lenses and micro-scratches. Finally, the Gaussian distribution of the collimated, expanded laser beam may introduce wavelength-dependent phase shifts that cause slight color fringing at the corners.

On the algorithmic side, the input data required by the OLAS method is a light field with depth information; while the algorithm stills works without accurate depth as seen in Figure 9, the accuracy suffers. This precludes the use of captured light fields for hologram generation, though running a depth estimation algorithm on the light field data, as done by Ziegler et al. [2007] for their light-fieldto-hologram transform, would be a viable option. Another option is to adapt the OLAS method to create a depth-free variant. In order for adjacent hogel shifts to constructively overlap and add in this case, the phase factor required would simply be based on the relative phase shift for each spatial frequency from one pixel to the next. This depth-free OLAS (dfOLAS) would be to the OLAS what the HS is to the PAS. The dfOLAS could also be used to encode specularities and other view-dependent effects with poorly defined depth (see supplemental Figure S3 for an example of the OLAS handling arbitrary view-dependent effects).

As noted in Section 4.1, the hogel size-invariance property has a minimum hogel size. The solution, while simple, is computationally expensive: render a light field with a large number of angular views to support a larger hogel size. A computationally less expensive solution may be to apply depth-of-field blur to antialias the light field (see supplement). The antialiasing can smooth out the point spread function of the objects in the hologram to improve visual quality, but cannot recover view-dependent effects that were not present in the low resolution light field.

Finally, on the topic of computational efficiency, the OLAS necessarily requires more compute than other HS algorithms due to the increase in density from tiling to per-pixel overlap, with the increase in cost proportional to the number of pixels per hogel. When comparing the runtime of MATLAB implementations of these algorithms for a $720 \times 1280$ phase image and a $9 \times 9$ hogel size, our implementation of the HS takes $2.04 \mathrm{sec}$ as compared to $32.7 \mathrm{sec}$ for the OLAS to compute the stereogram from the light field. However, an implementation of the OLAS in Python ran in $58.5 \mathrm{~ms}$ when run on a GPU (NVIDIA Quadro RTX 8000). Including the time for an extra propagation to the SLM, it takes $81.8 \mathrm{~ms}$ (compared with 26 and $28 \mathrm{sec}$ on CPU, respectively, using Python). Therefore, with sufficient parallelization, the increase in number of hogels may only have negligible effect on final runtime. Moreover, given that the OLAS has an effectively fixed hogel size and the computation for each hogel is nearly identical-which are often obstacles to efficient implementation of the Fresnel method on depth-varying scenes - it may be more readily optimized into dedicated hardware chips.

Code for the OLAS method is available on GitHub at https://github.com/computational-imaging/olas.

\section{CONCLUSION}

Holography shows great promise as a near-eye display technology for use in augmented and virtual reality applications. However, CGH algorithms have traditionally struggled to strike the balance between computation and high-quality reconstruction of the hologram. We develop a new approach to holographic stereograms that enables depth-independent high-resolution hologram generation. Furthermore, HS algorithms can start with a light field as the input data, allowing them to natively support view-dependent lighting effects. Our new algorithm, the overlap-add stereogram, therefore has characteristics that make it well suited for generation of complex scenes and promises to be a platform for the develop of further efficient light-field-to-hologram algorithms.

\section{ACKNOWLEDGMENTS}

The authors would like to thank Jane Xia for work on the first version of the hardware prototype and Ryan Spicer from Raxium for providing a Unity plugin we used to generate our scenes. This project was generously supported by funding from the National Science Foundation (NSF, award numbers 1553333 and 1839974), a Sloan Fellowship, an Okawa Research Grant, and Intel.

\section{REFERENCES}

J. Allen. 1977. Short term spectral analysis, synthesis, and modification by discrete Fourier transform. IEEE Trans. ASSP 25, 3 (1977), 235-238.

Martin J Bastiaans. 1978. The Wigner distribution function applied to optical signals and systems. Optics communications 25, 1 (1978), 26-30.

Stephen A. Benton. 1983. Survey Of Holographic Stereograms. In Proc. SPIE, Vol. 0367. Stephen A. Benton and V. Michael Bove. 2008. Holographic Imaging. Wiley-Interscience.

CB Burckhardt. 1970. A simplification of Lee's method of generating holograms by computer. Applied optics 9, 8 (1970), 1949-1949.

Jen-Hao Rick Chang, BVK Kumar, and Aswin C Sankaranarayanan. 2018. Towards multifocal displays with dense focal stacks. In SIGGRAPH Asia 2018 Technical Papers. ACM, 198.

Jhen-Si Chen and Daping Chu. 2015. Improved layer-based method for rapid hologram generation and real-time interactive holographic display applications. OSA Opt. Express 23, 14 (2015), 18143-18155.

Rick H-Y Chen and Timothy D Wilkinson. 2009. Computer generated hologram with geometric occlusion using GPU-accelerated depth buffer rasterization for threedimensional display. Applied optics 48, 21 (2009), 4246-4255.

Rainer G Dorsch, Adolf W Lohmann, and Stefan Sinzinger. 1994. Fresnel ping-pong algorithm for two-plane computer-generated hologram display. OSA Applied optics 33, 5 (1994), 869-875.

James R Fienup. 1978. Reconstruction of an object from the modulus of its Fourier transform. Optics letters 3, 1 (1978), 27-29.

James R Fienup. 1982. Phase retrieval algorithms: a comparison. Applied optics 21, 15 (1982), 2758-2769.

Qiankun Gao, Juan Liu, Jian Han, and Xin Li. 2016. Monocular 3D see-through headmounted display via complex amplitude modulation. OSA Opt. Express 24, 15 (2016), 17372-17383.

Ralph W Gerchberg. 1972. A practical algorithm for the determination of phase from image and diffraction plane pictures. Optik 35 (1972), 237-246.

Joseph W Goodman. 2005. Introduction to Fourier optics. Roberts and Company Publishers.

Joseph W. Goodman. 2014. Holography Viewed from the Perspective of the Light Field Camera. In Fringe 2013, Wolfgang Osten (Ed.). Springer Berlin Heidelberg, 3-15.

Joonku Hahn, Hwi Kim, Yongjun Lim, Gilbae Park, and Byoungho Lee. 2008. Wide viewing angle dynamic holographic stereogram with a curved array of spatial light modulators. OSA Opt. Express 16, 16 (2008), 12372-12386. 
Stephen Hamann, Liang Shi, Olav Solgaard, and Gordon Wetzstein. 2018. Timemultiplexed light field synthesis via factored Wigner distribution function. OSA Opt. Lett. 43, 3 (2018), 599-602.

R Häussler, S Reichelt, N Leister, E Zschau, R Missbach, and A Schwerdtner. 2009. Large real-time holographic displays: from prototypes to a consumer product. In Proc Stereoscopic Displays and Applications, Vol. 7237.

Keehoon Hong, Soon-gi Park, Jiwoon Yeom, Jonghyun Kim, Ni Chen, Kyungsuk Pyun, Chilsung Choi, Sunil Kim, Jungkwuen An, Hong-Seok Lee, et al. 2013. Resolution enhancement of holographic printer using a hogel overlapping method. Optics express 21, 12 (2013), 14047-14055.

CK Hsueh and AA Sawchuk. 1978. Computer-generated double-phase holograms. Applied optics 17, 24 (1978), 3874-3883.

Hong Hua and Bahram Javidi. 2014. A 3D integral imaging optical see-through headmounted display. Optics express 22, 11 (2014), 13484-13491.

Fu-Chung Huang, Kevin Chen, and Gordon Wetzstein. 2015. The light field stereoscope: immersive computer graphics via factored near-eye light field displays with focus cues. ACM Trans. Graph. (SIGGRAPH) 34, 4 (2015), 60.

Kishore Jaganathan, Yonina C Eldar, and Babak Hassibi. 2015. Phase retrieval: An overview of recent developments. arXiv preprint arXiv:1510.07713 (2015).

Changwon Jang, Kiseung Bang, Gang Li, and Byoungho Lee. 2018. Holographic Neareye Display with Expanded Eye-box. ACM Trans. Graph. (SIGGRAPH Asia) 37, 6 (2018).

Changwon Jang, Kiseung Bang, Seokil Moon, Jonghyun Kim, Seungjae Lee, and Byoungho Lee. 2017. Retinal 3D: augmented reality near-eye display via pupil-tracked light field projection on retina. ACM Trans. Graph. (SIGGRAPH Asia) 36, 6 (2017), 190.

Hoonjong Kang, Elena Stoykova, and Hiroshi Yoshikawa. 2016. Fast phase-added stereogram algorithm for generation of photorealistic 3D content. Applied optics 55, 3 (2016), A135-A143.

Hoonjong Kang, Takeshi Yamaguchi, and Hiroshi Yoshikawa. 2008. Accurate phaseadded stereogram to improve the coherent stereogram. OSA Appl. Opt. 47, 19 (2008).

Douglas Lanman, Matthew Hirsch, Yunhee Kim, and Ramesh Raskar. 2010. Contentadaptive parallax barriers: optimizing dual-layer 3D displays using low-rank light field factorization. ACM Trans. Graph. (SIGGRAPH Asia) 29, 6 (2010), 163:1-163:10.

Douglas Lanman and David Luebke. 2013. Near-eye light field displays. ACM Trans. Graph. (SIGGRAPH Asia) 32, 6 (2013), 220.

Douglas Lanman, Gordon Wetzstein, Matthew Hirsch, Wolfgang Heidrich, and Ramesh Raskar. 2011. Polarization Fields: Dynamic Light Field Display Using Multi-layer LCDs. ACM Trans. Graph. 30, 6, Article 186 (2011), 186:1-186:10 pages.

Seungjae Lee, Youngjin Jo, Dongheon Yoo, Jaebum Cho, Dukho Lee, and Byoungho Lee. 2019. Tomographic near-eye displays. Nature Communications 10, 1 (2019), 2497.

Wai Hon Lee. 1970. Sampled Fourier transform hologram generated by computer Applied Optics 9, 3 (1970), 639-643.

Marc Levoy and Pat Hanrahan. 1996. Light Field Rendering. In Proc. SIGGRAPH. 31-42.

Gang Li, Dukho Lee, Youngmo Jeong, Jaebum Cho, and Byoungho Lee. 2016. Holographic display for see-through augmented reality using mirror-lens holographic optical element. OSA Opt. Lett. 41, 11 (2016), 2486-2489.

Adolf W. Lohmann, Rainer G. Dorsch, David Mendlovic, Zeev Zalevsky, and Carlos Ferreira. 1996. Space-bandwidth product of optical signals and systems. OSA f. Opt. Soc. Am. A 13, 3 (1996), 470-473.

Mark Lucente. 1994. Diffraction-Specific Fringe Computation for Electro-Holography. Ph.D. Dissertation. Massachusetts Institute of Technology.

Mark Lucente and Tinsley A Galyean. 1995. Rendering interactive holographic images. In Proceedings of the 22nd annual conference on Computer graphics and interactive techniques. ACM, 387-394.

Andrew Maimone, Andreas Georgiou, and Joel S Kollin. 2017. Holographic near-eye displays for virtual and augmented reality. ACM Transactions on Graphics (TOG) 36 , 4 (2017), 85 .

Kyoji Matsushima and Sumio Nakahara. 2009. Extremely high-definition full-parallax computer-generated hologram created by the polygon-based method. Applied optics 48, 34 (2009), H54-H63.

Eunkyong Moon, Myeongjae Kim, Jinyoung Roh, Hwi Kim, and Joonku Hahn. 2014 Holographic head-mounted display with RGB light emitting diode light source. OSA Opt. Express 22, 6 (2014), 6526-6534.

Jae-Hyeung Park. 2017. Recent progress in computer-generated holography for threedimensional scenes. Fournal of Information Display 18, 1 (2017), 1-12.

Jae-Hyeung Park and Mehdi Askari. 2019. Non-hogel-based computer generated hologram from light field using complex field recovery technique from Wigner distribution function. Optics express 27, 3 (2019), 2562-2574.

Yifan Peng, Xiong Dun, Qilin Sun, and Wolfgang Heidrich. 2017. Mix-and-match holography. ACM Trans. Graph. 36, 6 (2017), 191

Kishore Rathinavel, Hanpeng Wang, Alex Blate, and Henry Fuchs. 2018. An extended depth-at-field volumetric near-eye augmented reality display. IEEE transactions on visualization and computer graphics 24, 11 (2018), 2857-2866.

Pasi Saarikko and Pasi Kostamo. 2016. Waveguide. US Patent 9,513,480 B2.
Brian Schowengerdt, Dianmin Lin, and Pierre St. Hillaire. 2018. Waveguide. US Patent Application 0,052,277 A1.

Liang Shi, Fu-Chung Huang, Ward Lopes, Wojciech Matusik, and David Luebke. 2017 Near-eye Light Field Holographic Rendering with Spherical Waves for Wide Field of View Interactive 3D Computer Graphics. ACM Trans. Graph. (SIGGRAPH Asia) 36, 6, Article 236 (2017), 236:1-236:17 pages.

Chris Slinger, Colin Cameron, and Maurice Stanley. 2005. Computer-generated holography as a generic display technology. Computer 38, 8 (2005), 46-53.

Quinn YJ Smithwick, James Barabas, Daniel E Smalley, and V Michael Bove Jr. 2010. Interactive holographic stereograms with accommodation cues. In Practical Holography XXIV: Materials and Applications, Vol. 7619. International Society for Optics and Photonics, 761903.

Koki Wakunami, Hiroaki Yamashita, and Masahiro Yamaguchi. 2013. Occlusion culling for computer generated hologram based on ray-wavefront conversion. Optics express 21, 19 (2013), 21811-21822.

Gordon Wetzstein, Douglas Lanman, Wolfgang Heidrich, and Ramesh Raskar. 2011. Layered 3D: Tomographic Image Synthesis for Attenuation-based Light Field and High Dynamic Range Displays. ACM Trans. Graph. 30, 4, Article 95 (2011), 95:195:12 pages.

Gordon Wetzstein, Douglas Lanman, Matthew Hirsch, and Ramesh Raskar. 2012. Ten sor Displays: Compressive Light Field Synthesis using Multilayer Displays with Directional Backlighting. ACM Trans. Graph. (SIGGRAPH) 31, 4 (2012), 1-11.

Masahiro Yamaguchi, Hideshi Hoshino, Toshio Honda, and Nagaaki Ohyama. 1993. Phase-added stereogram: calculation of hologram using computer graphics technique. In Practical Holography VII: Imaging and Materials, Vol. 1914. International Society for Optics and Photonics, 25-32.

Fahri Yaras, Hoonjong Kang, and Levent Onural. 2010. State of the Art in Holographic Displays: A Survey. Journal of Display Technology 6, 10 (2010), 443-454.

Toyohiko Yatagai. 1976. Stereoscopic approach to 3-D display using computer-generated holograms. Applied optics 15, 11 (1976), 2722-2729.

Han-Ju Yeom, Hee-Jae Kim, Seong-Bok Kim, HuiJun Zhang, BoNi Li, Yeong-Min Ji, Sang-Hoo Kim, and Jae-Hyeung Park. 2015. 3D holographic head mounted display using holographic optical elements with astigmatism aberration compensation. OSA Opt. Express 23, 25 (2015), 32025-32034.

Hao Zhang, Liangcai Cao, and Guofan Jin. 2017. Computer-generated hologram with occlusion effect using layer-based processing. Applied optics 56, 13 (2017), F138F143.

Hao Zhang, Neil Collings, Jing Chen, Bill A Crossland, Daping Chu, and Jinghui Xie. 2011. Full parallax three-dimensional display with occlusion effect using computer generated hologram. Optical Engineering 50, 7 (2011), 074003.

Hao Zhang, Yan Zhao, Liangcai Cao, and Guofan Jin. 2015. Fully computed holographic stereogram based algorithm for computer-generated holograms with accurate depth cues. Optics express 23, 4 (2015), 3901-3913.

Zhengyun Zhang and M. Levoy. 2009. Wigner distributions and how they relate to the light field. In Proc. ICCP. 1-10.

Zichen Zhang, Zheng You, and Daping Chu. 2014. Fundamentals of phase-only liquid crystal on silicon (LCOS) devices. Light: Science \& Applications 3 (2014), e213.

Remo Ziegler, Simon Bucheli, Lukas Ahrenberg, Marcus Magnor, and Markus Gross. 2007. A Bidirectional Light Field-Hologram Transform. In Computer Graphics Forum (Eurographics), Vol. 26. 435-446.

Matthias Zwicker, Wojciech Matusik, Fredo Durand, and Hans-Peter Pfister. 2006. Antialiasing for automultiscopic 3D displays. In Eurographics Symposium on Rendering.

\section{APPENDIX}

In this section, we briefly review the mathematical foundations of holographic near-eye display optics, which are essential to correctly rendering the light fields to be used as inputs to the OLAS such that it produces the correct images for our near-eye display prototype.

We consider an optical setup that uses a coherent light source, i.e. a laser, which is collimated by a lens (see Figure 11). The resulting plane wave is incident on a spatial light modulator (SLM) where diffraction occurs. Diffraction is caused by the SLM modulating amplitude and/or phase of the incident plane wave in a spatially varying manner. Phase-only SLMs are most commonly used in holographic displays, so we also consider this type of SLM in the following. Due to the fact that a phase-only SLM cannot modulate the amplitude of the incident light, it solely relies on the perceived interference of the diffracted light to create a spatially varying 


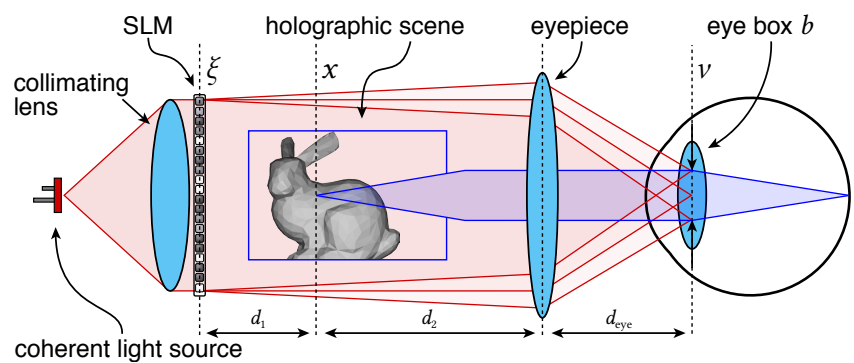

Fig. 11. Illustration of a holographic near-eye display. Collimated laser light is incident on a spatial light modulator (SLM). The diffracted light creates an interference pattern that is perceived as a scene by the user. The scene is magnified by an eyepiece, but it is only visible over a limited eyebox. Illumination light paths are shown in red and detection paths in blue.

intensity distribution at some distance in front of it, i.e. a target image.

An interesting property of holographic displays is that their image resolution is only indirectly defined by the SLM resolution. In this context, we consider image resolution to be the lateral and axial spot sizes $r_{\ell}$ and $r_{a}$ at a distance $d_{1}$ that can be produced by displaying an appropriate phase pattern on the SLM. The diffraction-limited spot sizes are defined by Abbe's law as $r_{\ell}=\lambda /(2 \mathrm{NA})$ and $r_{a}=2 \lambda /\left(\mathrm{NA}^{2}\right)$, where $\lambda$ is the wavelength. NA $=n \sin \left(\theta_{\max }\right)$ is the numerical aperture, where $n$ being the refractive index of the medium $\left(n_{\text {air }} \approx 1\right)$ and $\theta_{\max }$ being the maximum diffraction angle of the SLM. This diffraction angle can be calculated given the SLM pixel size $p$ via the grating equation as $\theta_{\max }=\sin ^{-1}(\lambda /(2 p))$. Thus, the lateral spot size would be $r_{\ell}=p$ but in practice, the NA is also limited by the size of the SLM, $w$, resulting in

$$
\begin{aligned}
& r_{\ell}=\frac{\lambda}{2 \mathrm{NA}} \approx \max \left(p, \frac{d_{1} \lambda}{w}\right) \\
& r_{a}=\frac{2 \lambda}{\mathrm{NA}^{2}} \approx \max \left(\frac{8 p^{2}}{\lambda}, \frac{8 \lambda d_{1}^{2}}{w^{2}}\right) .
\end{aligned}
$$

While Equation 5 applies to any holographic display, a near-eye display usually has an eyepiece, i.e. a lens with focal length $f$, to magnify the image. Assuming that the holographic image is located at a distance $d_{2}$ away from the eyepiece, the distance between eyepiece and magnified virtual image is given by the Gaussian thin lens formula as $d_{v}=\left|1 /\left(\frac{1}{f}-\frac{1}{d_{2}}\right)\right|$ and the magnification is $M=f /\left(f-d_{2}\right)$. Thus, given the eye relief distance $d_{\text {eye }}$, we can calculate the field of view of the near-eye display as

$$
\mathrm{FOV}=2 \tan ^{-1}\left(\frac{M w}{2\left(d_{v}+d_{\text {eye }}\right)}\right) \approx 2 \tan ^{-1}\left(\frac{w}{2 f}\right) .
$$

The approximation in the right part of Equation 6 is valid for the special case $d_{2}=d_{\text {eye }}=f$. While this case seems restrictive, it makes a general fact intuitive: eyepieces with longer focal lengths offer smaller fields of view and vice versa. The size of the eyebox $b$, over which the user can see the holographic scene, is

$$
b=2 d_{\text {eye }} \tan \left(\theta_{\max }\right) \approx d_{\text {eye }} \frac{\lambda}{p}
$$

where the approximation is valid in the paraxial domain, i.e. $x \approx$ $\sin x \approx \tan x$.

Equations 5-7 reveal two dilemmas of holographic near-eye displays: (i) the space-bandwidth product offered by current-generation SLMs is a major limiting factor for resolution, depth of field, field of view, and eyebox of holographic displays and (ii) there is a fundamental tradeoff between field of view and eyebox size in most near-eye displays known as etendue.

The space-bandwidth product of an SLM refers to the tradeoff between its size $w$ and bandwidth, i.e. the range of spatial frequencies $u$ (or angles $\theta$, where $u=\sin (\theta) / \lambda$ ) that are addressable with a finite pixel size $p$ [Lohmann et al. 1996]. To maximize the resolution, depth of field, and field of view of any holographic display, we require large SLMs with small pixel sizes (on the order of the wavelength). Unfortunately, current SLMs offer pixel sizes in the order of a few microns but even for resolutions of $4 \mathrm{~K}$ and beyond, the SLM is only a few millimeters in size. Tiling multiple SLMs is one solution to extending the space-bandwidth product but it requires a massive amount of data processing and significantly increases the alignment complexity of such a display [Hahn et al. 2008; Slinger et al. 2005].

Etendue is a closely related concept that we consider a characteristic of the eyepiece in a near-eye display. One can choose an eyepiece with a short focal length to maximize the field of view, but this choice decreases the size of the eyebox proportionally (Equations 6-7). Approaches to overcoming the limits set by etendue are commonly referred to as exit pupil expansion. Two prominent examples include exit pupil replication and pupil steering. For exit pupil replication, sophisticated eyepieces create multiple copies of the exit pupil such that the eyebox is filled with these non-overlapping copies [Saarikko and Kostamo 2016; Schowengerdt et al. 2018]. This approach increases the eyebox size at the cost of light efficiency and is likely used by commercial devices such as Microsoft's HoloLens and Magic Leap's ML1. Pupil steering is enabled by eye tracking and allows a small exit pupil to be optically steered to create a larger virtual eyebox [Häussler et al. 2009; Jang et al. 2018, 2017] at the cost of increased system complexity due to the need for beam steering and eye tracking.

ACM Trans. Graph., Vol. 38, No. 6, Article 214. Publication date: November 2019. 